\title{
Therapeutic intervention for genetic disease by the augmented recycling of target proteins
}

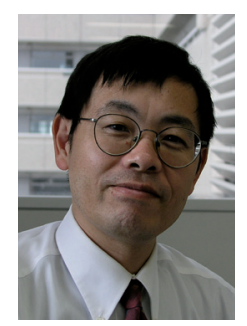

\author{
"Prevention of protein or mRNA degradation \\ is a potential therapeutic intervention in diseases \\ resulting from haploinsufficiency, \\ including lissencephaly."
}

\section{Masami Yamada, Shinji Hirotsune ${ }^{\dagger}$ \& Anthony Wynshaw-Boris}

†Author for correspondence: Department of Genetic Disease Research, Osaka City University Graduate School of Medicine Asahi-machi 1-4-3 Abeno, Osaka 545-8585, Japan w Tel.: +81 666453725 = Fax: +81666453727 m shinjih@med.osaka-cu.ac.jp

Lissencephaly is a severe human neuronal migration defect characterized by a smooth cerebral surface, thick cortex and dilated lateral ventricules, associated with mental retardation and seizures [1-3]. LIS1 was the first gene cloned in an organism that was found to be important for neuronal migration, as it was deleted or mutated in patients with lissencephaly in a heterozygous fashion [4]. Studies in model organisms, particularly Aspergillus nidulans [5,6], as well as those in the mouse [7], have uncovered an evolutionarily conserved pathway that involves LIS1 and cytoplasmic dynein. Cytoplasmic dynein is a highly complex molecular motor that transports its attached cargo towards the minus-end of microtubules. These enzymes are required for many essential motile activities within the cytoplasm [8-10]. However, until recently the underlying mechanism by which LIS1 regulated cytoplasmic dynein was unknown. We recently reported that LIS1 holds cytoplasmic dynein on microtubules in an 'idling state', thereby making a stable complex of microtubule-LIS1-cytoplasmic dynein [11]. This microtubule-LIS1-cytoplasmic dynein complex is thought to be transported to the plus-end of microtubules by kinesin, which is essential for the maintainence of proper cytoplasmic dynein distribution within the cell. Interestingly, a substantial fraction of LIS1 is degraded by the cystein protease, calpain, after reaching the plus-end of microtubules. This gave us an idea: could the levels of LIS1 protein be increased by inhibiting the calpainmediated degradation of LIS1 at the cortex of the cell? If we were able to increase the levels of LIS1 protein in this fashion, would this result in any therapeutic benefit? It turns out, using genetic mouse models with reduced levels of LIS1, that the answer to both questions is yes.

In a recently published study, we demonstrated that inhibition of calpain restored LIS1 protein to a normal level using mouse embryonic fibroblast cells or dorsal root ganglia neurons established from $\mathrm{Lis}^{\mathrm{H}^{+-}}$mice [12]. We further demonstrated that inhibition of calpain led to the rescue of the aberrant distribution of cytoplasmic dynein and intracellular components, including mitochondria and $\beta$-coat protein-positive vesicles in Lis $^{+/-}$mouse embryonic fibroblast cells, and improved neuronal migration of $L i s 1^{+/-}$cerebellar granular neurons. Intraperitoneal injection of a calpain inhibitor to pregnant $\mathrm{Lis}^{+/-}$dams rescued apoptotic neuronal cell death, reduction of brain weight and neuronal migration defects in Lis $^{+/-}$offspring. Thus, we used our knowledge of the pathogenesis and mechanism of action of LIS1 and its pathway to directly assess the effect of inhibition of calpain with quantitative phenotypic measures in vivo and in vitro.

\footnotetext{
"Studies in model organisms have uncovered an evolutionarily conserved pathway that involves LIS1 and cytoplasmic dynein."
}

This approach provides proof-of-principle for a potential therapeutic intervention to a severe developmental cortical dysplasia, lissencephaly. Although extensive work has been performed in order to understand the molecular and cellular biological basis of lissencephaly due to haploinsufficiency of LIS1, therapeutic strategies for lissencephaly have not been addressed. This is 
probably because the treatment of lissecephaly is a daunting task for several reasons. First, in lissencephaly, early brain development is affected, and so treatment would be necessary throughout development. Second, all neurons throughout the brain would have to be treated, since lissencephaly results in global defects in brain development. Third, LIS1 mutations in humans are de novo, presenting a problem of detection at an early enough time point to allow therapy to commence when it can still make a difference. Despite these difficulties, there are some advantages to considering how to treat lissencephaly resulting from LIS1 haploinsufficiency. First, LIS1 protein is present because individuals have heterozygous loss of LIS1; therefore, one can consider augmenting the already present but low levels of LIS1 protein in affected individuals. In addition, there are dosage-dependent effects of LIS1, and so any augmentation of LIS1 protein levels will probably have a beneficial effect. Finally, a great deal is known about the pathogenesis and mechanism of action of LIS1 and its pathway; therefore, the effects of any therapeutic modality can be assessed directly with quantitative measures in vivo and in vitro.

"...calpain inhibitors hold promise for the
treatment of neuromuscular and
neurodegenerative diseases in which
calpains have been shown to be
upregulated, for example Parkinson's
disease and Duchenne
muscular dystrophy."

Modulation of calpain activity has already been attampted therapeutically. The calpains are a class of cellular cysteine proteases that require calcium and are functionally active at neutral $\mathrm{pH}[13,14]$. Calpain activation can take place in two modes: controlled activation under physiological conditions, and hyperactivation under pathological conditions that involve sustained calcium overload. The known calpain inhibitors are small molecule compounds, suggesting that it is possible to deliver calpain inhibitors to the target tissue. The major first-generation calpain inhibitors tested as anticataract agents were the epoxysuccinyl peptides, which include E64 and its analogs. These inhibitors have electrophilic centers within their structure that form an irreversible covalent bond with the thiol group of the cysteine residue in the active site of calpain, thereby blocking its proteolytic action. Structural modification of E64 produced E64d, which showed improved membrane permeability and is readily hydrolyzed to an active form by lens enzymes in vivo. Moreover, a significant impetus to these approaches was provided by the recently solved structures for calpain-2 [15,16]. The detailed structural and mechanistic information gleaned from these structures opened the door to structure-based calpain inhibitor design, and, in this capacity, led to the synthesis of mercapto acrylate derivatives. One promising compound is SNJ-1945 [17-19]. SNJ-1945 is highly permeable in the CNS, which was demonstrated by its effects on retinal or neuronal protection. Moreover, SNJ-1945 showed a high selectivity for calpains, with no significant effect on the activity of many other enzymes, such as inhibition of cysteine proteases and serine proteases, which reduced the viability of previously tested calpain inhibitors. Therefore, the usage of newgeneration calpain inhibitors may provide a more feasible approach for therapeutic intervention in human disease.

Besides our study using calpain inhibition to partially rescue the neuronal migration phenotype of Lis1 mutant mice, it was recently demonstrated that postnatal conditional expression of DCX, the protein product of an X-linked gene for lissencephaly, can reduce heterotopia and restore neuronal patterning in animal models [20]. Both laminar displacement of neurons and the size of subcortical band heterotopia are reduced upon delayed expression of DCX during early postnatal periods. These encouraging findings suggest that cortical neurons maintain competence for migration after birth, and that treatment of lissencephaly after birth from perinatal stages may also provide some therapeutic benefits for the treatment of lissencephaly. In such cases, a drug that can pass the BBB will be required. Penetration of the $\mathrm{BBB}$ by SNJ-1945 has previously been demonstrated, making it a good choice for potential therapeutic trials in neonates [19].

The use of protease inhibitors or other agents that can increase protein levels may be generally applicable as therapeutic approaches for other genetic disorders that result from haploinsufficiency. Haploinsufficiency is defined as a dominant phenotype in diploid organisms that are heterozygous for a loss-of-function allele. The total level of a gene product (a particular protein) produced by the cell is approximately half of the normal level, which is not sufficient to permit the cell to function normally. Many of the haploinsufficient mutations in humans are observed in transcription factors [21]. For example, mutations 
in the gene encoding for the CREB-binding protein (CBP) result in Rubinstein-Taybi syndrome, a rare condition that occurs in one out of 125,000 births and is characterized by mental retardation and skeletal abnormalities [22]. Loss of function of $C B P$ may therefore interfere with the transcriptional activation of target genes in two ways: by preventing recruitment to the promoter of the basal transcription machinery and by blocking chromatin remodeling. Marfan syndrome and Ehlers-Danlos syndrome type IV are clinical entities characterized by vascular abnormalities that result from mutations of structural components of the extracellular matrix - another example of haploinsufficiency [23,24]. The relevance of haploinsufficiency in human disease has become increasingly apparent, and any human diseases that result from haploinsufficiency are potential targets of our strategy to boost levels of the haploinsufficient protein. Prevention of protein degradation or inhibition of mRNA degradation will be an effective method to increase levels of mutated protein in each disease. In addition to treatment of lissencephaly, calpain inhibitors hold promise for the treatment of neuromuscular and neurodegenerative diseases in which calpains have been shown to be upregulated, for example Parkinson's disease and Duchenne muscular dystrophy [25]. Application of calpain inhibitors to Duchenne muscular dystrophy might have a more direct effect via prevention of degradation of utrophin protein that has similar function with dystrophin [26]. Calpain inhibitors might prevent the degradation of utrophin, which may then ameliorate the progression of muscular wasting. Sporadic morbidity to neurodegenerative diseases, such as Parkinson's disease or Alzheimer's disease, might be attributable to hemizygous mutations of responsible genes. Prevention of protein or mRNA degradation will also be a potential strategy for therapeutic intervention in such cases.

\section{Acknowledgements \\ The authors thank Yoshibiko Funae, Hiroshi Iwao, Toshio Yamauchi, Masami Muramatsu, Yoshitaka Nagai and Michiyuki Matsuda for generous support and encouragement.}

\section{Financial \& competing interests disclosure \\ This work was supported by Grant-in-Aid for Scientific Research from the Ministry of Education, Science, Sports and Culture of Japan from the Ministry of Education, Science, Sports and Culture of Japan to Shinji Hirotsune. This work was also supported by The Mother and Child Health Foundation, The Naito Foundation, Japan Brain Foundation and The Uehara Memorial Foundation to Shinji Hirotsune, and NIH grants NS41030 and HD47380 to Anthony Wynshaw- Boris. The authors have no other relevant affliations or financial involvement with any organization or entity with a financial interest in or financial conflict with the subject matter or materials discussed in the manuscript apart from those disclosed. \\ No writing assistance was utilized in the production of this manuscript.}

\section{Bibliography}

1. Dobyns WB: The neurogenetics of lissencephaly. Neurol. Clin. 7, 89-105 (1989).

2. Dobyns WB, Curry CJ, Hoyme HE, Turlington L, Ledbetter DH: Clinical and molecular diagnosis of Miller-Dieker syndrome. Am. J. Hum. Genet. 48, 584-594 (1991).

3. Wynshaw-Boris A: Lissencephaly and LIS1: insights into the molecular mechanisms of neuronal migration and development. Clin. Genet. 72, 296-304 (2007).

4. Reiner O, Carrozzo R, Shen Y et al.: Isolation of a Miller-Dieker lissencephaly gene containing $G$ protein $\beta$-subunit-like repeats. Nature 364, 717-721 (1993).

5. Morris NR, Efimov VP, Xiang X: Nuclear migration, nucleokinesis and lissencephaly. Trends Cell. Biol. 8, 467-470 (1998).

6. Morris SM, Albrecht U, Reiner O, Eichele G, Yu-Lee LY: The lissencephaly gene product Lis1, a protein involved in neuronal migration, interacts with a nuclear movement protein, NudC. Curr. Biol. 8, 603-606 (1998).

7. Hirotsune S, Fleck MW, Gambello MJ et al:: Graded reduction of Pafah1b1 (Lis1) activity results in neuronal migration defects and early embryonic lethality. Nat. Genet. 19, 333-339 (1998).

8. Vallee RB, Shpetner HS, Paschal BM: The role of dynein in retrograde axonal transport. Trends Neurosci. 12, 66-70 (1989).

9. Vallee RB, Tai C, Faulkner NE: LIS1: cellular function of a disease-causing gene. Trends Cell. Biol. 11, 155-160 (2001).

10. Vallee RB, Tsai JW: The cellular roles of the lissencephaly gene LISI, and what they tell us about brain development. Genes Dev. 20, 1384-1393 (2006).

11. Yamada M, Toba S, Yoshida Y et al.: LIS1 and NDEL1 coordinate the plus-end-directed transport of cytoplasmic dynein. Embo J. 27, 2471-2483 (2008).
12. Yamada M, Yoshida Y, Mori D et al.: Inhibition of calpain increases LIS1 expression and partially rescues in vivo phenotypes in a mouse model of lissencephaly. Nat. Med. 15(10), 1202-1207 (2009).

13. Goll DE, Thompson VF, Li H, Wei W, Cong J: The calpain system. Physiol. Rev. 83, 731-801 (2003).

14. Suzuki K, Hata S, Kawabata Y, Sorimachi H: Structure, activation, and biology of calpain. Diabetes 53(Suppl. 1), S12-S18 (2004).

15. Hosfield CM, Elce JS, Davies PL, Jia Z: Crystal structure of calpain reveals the structural basis for $\mathrm{Ca}^{2+}$-dependent protease activity and a novel mode of enzyme activation. Embo J. 18, 6880-6889 (1999).

16. Strobl S, Fernandez-Catalan C, Braun M et al: : The crystal structure of calcium-free human m-calpain suggests an electrostatic switch mechanism for activation by calcium. Proc. Natl Acad. Sci. USA 97, 588-592 (2000). 
17. Koumura A, Nonaka Y, Hyakkoku K et al.: A novel calpain inhibitor, ((1S)-1((((1S)-1benzyl-3-cyclopropylamino-2,3-di-oxopropyl) amino)carbonyl )-3-methylbutyl) carbamic acid 5-methoxy-3-oxapentyl ester, protects neuronal cells from cerebral ischemia-induced damage in mice. Neuroscience 157, 309-318 (2008).

18. Oka T, Walkup RD, Tamada Y et al.: Amelioration of retinal degeneration and proteolysis in acute ocular hypertensive rats by calpain inhibitor ((1S)-1-((((1S)-1-benzyl3-cyclopropylamino-2,3-di-oxopropyl) amino)carbony l)-3-methylbutyl)carbamic acid 5-methoxy-3-oxapentyl ester. Neuroscience 141, 2139-2145 (2006).

19. Shirasaki Y, Yamaguchi M, Miyashita H: Retinal penetration of calpain inhibitors in rats after oral administration. J. Ocul. Pharmacol. Ther. 22, 417-424 (2006).

20. LoTurco J, Manent JB, Sidiqi F: New and improved tools for in utero electroporation studies of developing cerebral cortex. Cereb. Cortex 19(Suppl. 1), I120-I125 (2009).
21. Seidman JG, Seidman C: Transcription factor haploinsufficiency: when half a loaf is not enough. J. Clin. Invest. 109, 451-455 (2002).

22. Petrij F, Giles RH, Dauwerse HG et al.: Rubinstein-Taybi syndrome caused by mutations in the transcriptional co-activator CBP. Nature 376, 348-351 (1995).

23. Dietz HC, Cutting GR, Pyeritz RE et al:: Marfan syndrome caused by a recurrent de novo missense mutation in the fibrillin gene. Nature 352, 337-339 (1991).

24. Toriello HV, Glover TW, Takahara K et al: A translocation interrupts the COL5A1 gene in a patient with Ehlers-Danlos syndrome and hypomelanosis of Ito. Nat. Genet. 13, 361-365 (1996).

25. Saez ME, Ramirez-Lorca R, Moron FJ, Ruiz A: The therapeutic potential of the calpain family: new aspects. Drug Discov. Today 11, 917-923 (2006).

26. Banks GB, Chamberlain JS: The value of mammalian models for duchenne muscular dystrophy in developing therapeutic strategies. Curr. Top. Dev. Biol. 84, 431-453 (2008).

\section{Affiliations}

- Masami Yamada

Department of Genetic Disease Research, Osaka City University Graduate School of Medicine Asahi-machi 1-4-3 Abeno, Osaka 545-8585, Japan

Tel.: +81666 453726 my26@med.osaka-cu.ac.jp

- Shinji Hirotsune

Department of Genetic Disease Research, Osaka City University Graduate School of Medicine Asahi-machi 1-4-3 Abeno, Osaka 545-8585, Japan

Tel.: +81666 453725

Fax: +81666453727 shinjih@med.osaka-cu.ac.jp

- Anthony Wynshaw-Boris UCSF School of Medicine, Department of Pediatrics and Institute for Human Genetics, San Francisco, CA 94143, USA Tel.: +1 4154765184 wynshawborist@peds.ucsf.edu 